\title{
Distributed Quality System under the Condition of Wireless Network
}

\author{
Mengqing Tanli ${ }^{1}$, Yan Jiang ${ }^{1}$, Rushu Peng ${ }^{1}$, Chunliang Zhang ${ }^{1}$ \\ 1- School of Mech. \& Eng., University of South China, Hengyang, China 421001, \\ e-mail:TLMQ-TEAM@163.com,TLMQPrivate@tom.com
}

\begin{abstract}
Comparison of wireless broadband and cable network is analyzed firstly, and models of distributed quality system under the condition of wireless network are put forward, and core technologies are discussed. The hierarchy model can be divided into four layers: device layer, disposal layer, control layer, strategies management layer, and main functions include: quality research and new product testing, quality system and standardization audit, quality planning, procurement quality control, process quality control, product quality inspection, assembly sample testing, product measurement and experiment, quality information statistics. The core technologies include: digital detection technology, the quality data and information online interaction technology, CAQ mailbox technology, wireless network construction technology, data backup strategies and security technology.
\end{abstract}

Keywords- Intranet/Extranet, wireless broadband, quality system

\section{COMPARISON OF WIRELESS AND WIRED NETWORK}

Intranet/Extranet concepts generated in the late of 20th century, which is the development of Internet technology in enterprise applications, and Intranet/Extranet technology based on the new wireless broadband technology breaks the distributed plant and workshop's information isolation in the past, realizes an information integration of the device, group, workshop, factory and external branches, and becomes a new technology of energy-saving and being movable.

Cable network for some time in the past accounted for considerable market share in the field of network applications, but network made up of fiber optic cable or other cable has essential inadequate: (a)wasted wiring and rewiring labor, (b)easily damaged line, (c)all sites in the network cannot be moved. Especially when two network are connected apart from a few kilometers to decades kilometers and the phone lines are taken as the transmission medium, there are some disadvantages such as low rate, high bit-error rate and poor reliability of line; Effective ways to resolve these difficulties are to use the latest wireless communications technologies, that is, wireless broadband technology. In general, wireless communication network has some advantages: (a)Laying out of the cable is not constraint by the geographical environment, set-up time is shorter, the cost is lower; (b) Need not to change original equipment; (c)Operating cost is lower; (d) Supporting for mobile. [1]

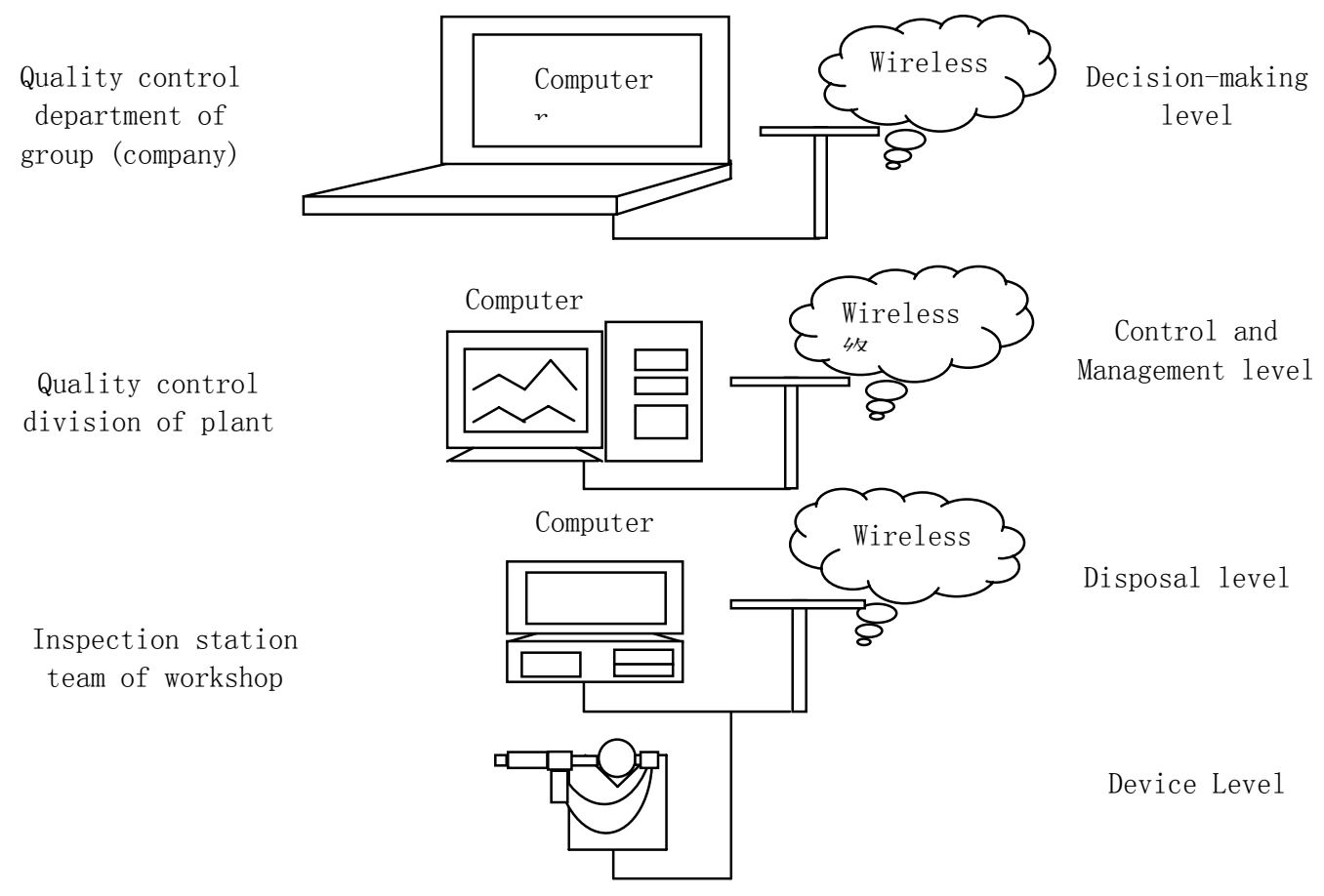

Figure 1. Hierarchical model of distributed quality system 


\section{THE HIERARCHY MODEL OF DISTRIBUTED QUALITY SYSTEM}

Distributed quality system under the condition of wireless network can be divided into four layers: the device layer, disposal layer, control and management layer, decision-making layer, the detail is shown in figure 1 . The device layer mainly includes testing instrument and detection device, which is the most basic material base of the system. The second layer, disposal layer is the work foundation of quality system, which is responsible to the parts sampling and inspection, to judge if it is qualified or not, then eliminate mistakes, and achieve the quality data collection, in this layer it have to guarantee that the quality data is accurate and reliable. The 3rd layer of quality system, which is responsible to analyze, control and correct the quality information submitted by the lower layers. Decision-making layer is the upper layer of quality system, which is responsible for quality planning, quality decision, quality supervision, and quality information checking, correcting and improving. [2-4]

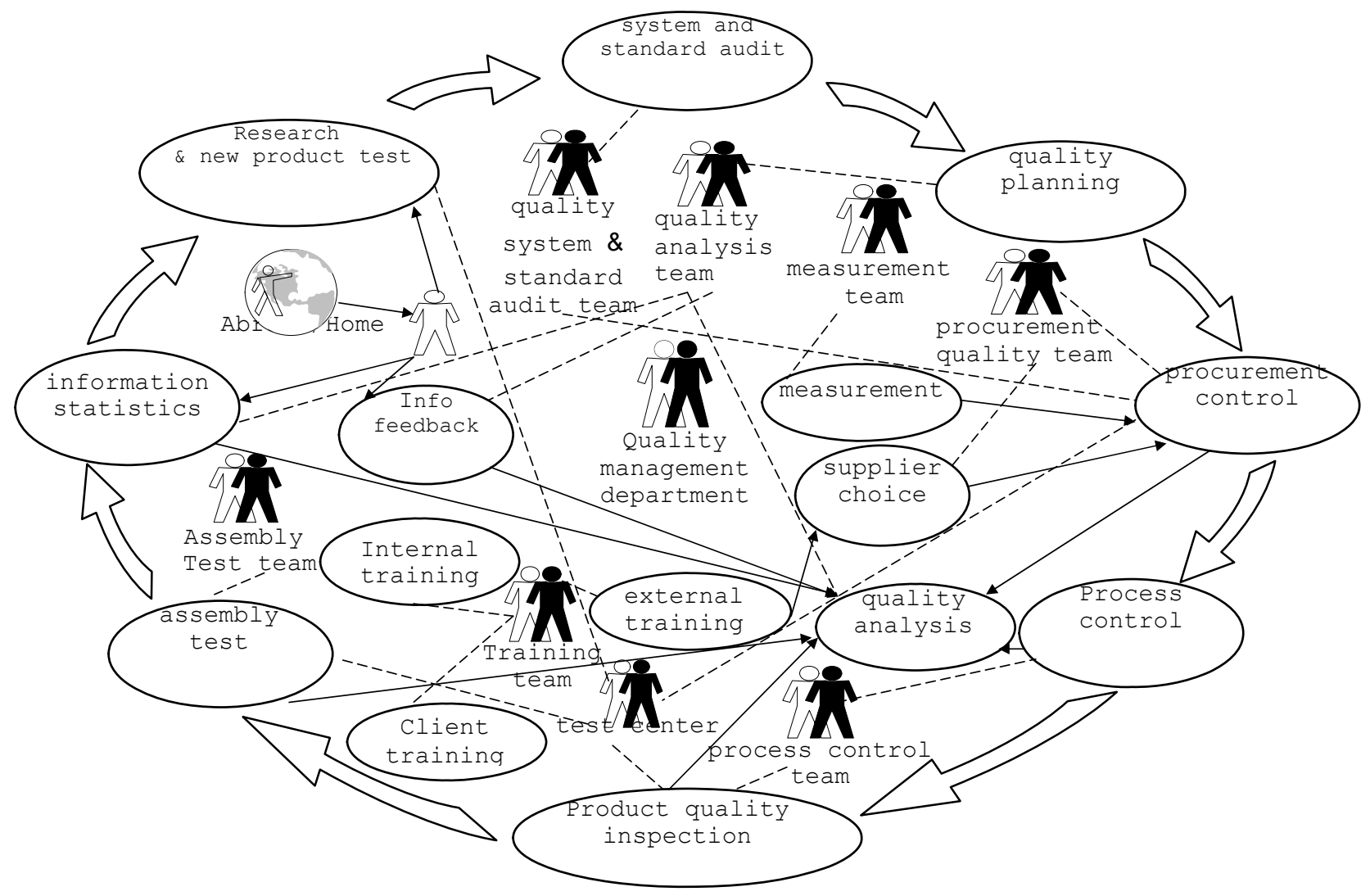

Figure 2. Occupation model of distributed quality system

\section{OCCUPATION MODEL OF DISTRIBUTED QUALITY SYSTEM}

Quality system under the condition of wireless network approximately has similar behavior against the past, with supporting of the overall enterprise strategies, all the behaviors which keep the enterprise quality system operate reliably are included. It mainly includes: quality research and new product test, quality system and standardization examination and audit, quality planning, procurement quality control, process quality control, product quality inspection, assembly sampling and test, product measurement and experiment, and quality information analysis and statistics. The detail of the model is shown in figure 2. [4-5]

\section{NETWORK STRUCTURE MODEL OF DISTRIBUTED QUALITY SYSTEM}

The general strategy of constructing wireless network of distributed quality system is that Intranet acts as the foundation, and Extranet acts for expanding, with Internet technology for support, to implement interconnection of the wireless broadband. Based on this principle, network structure of the distributed quality system can be divided into three parts: the local quality system (Intranet), branch quality system (Extranet) and external quality system (Extranet), the distributed quality system nets is shown in figure 3. [3-4] 


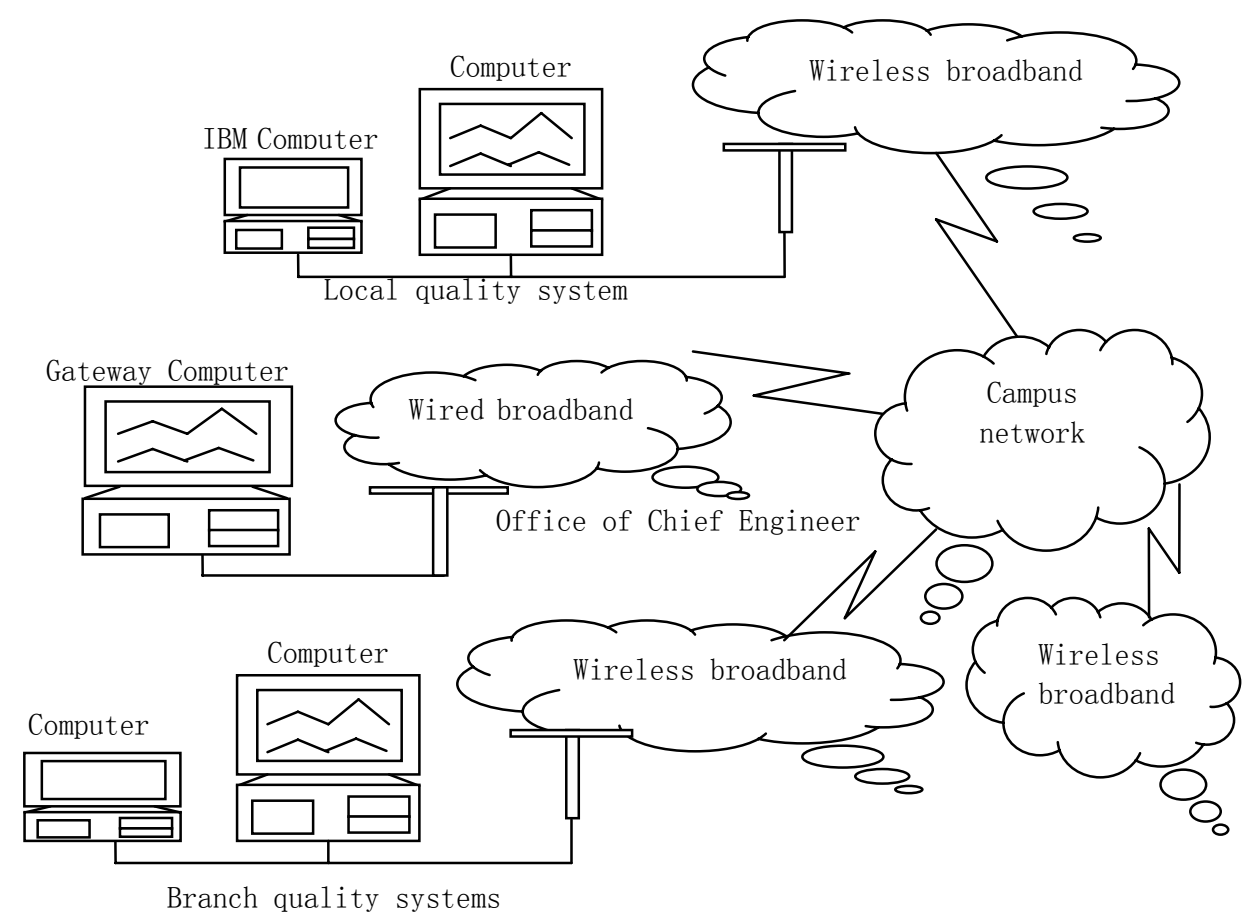

Figure 3. Network structure model of wireless network (experiment nets )

The implementation of experimental nets is carried on in University of South China, and local quality system interconnected by wireless network card, which was built in the laboratory, branch quality system was built in students' apartment buildings, and external quality system was set in the teachers' office, with campus network as interconnection pathway. By running the network, it proved that the network structure is reasonable, and the technical program is executable.

\section{THE CORE TECHNOLOGY OF DISTRIBUTED QUALITY SYSTEM}

Distributed quality system under the condition of wireless network, whose core technologies generally include: (a) digital detection technology; (b) quality data and datum online interactive technology; (c) CAQ mailbox technology; (d) wireless network construction technology; (e) data saving strategy and security technology.

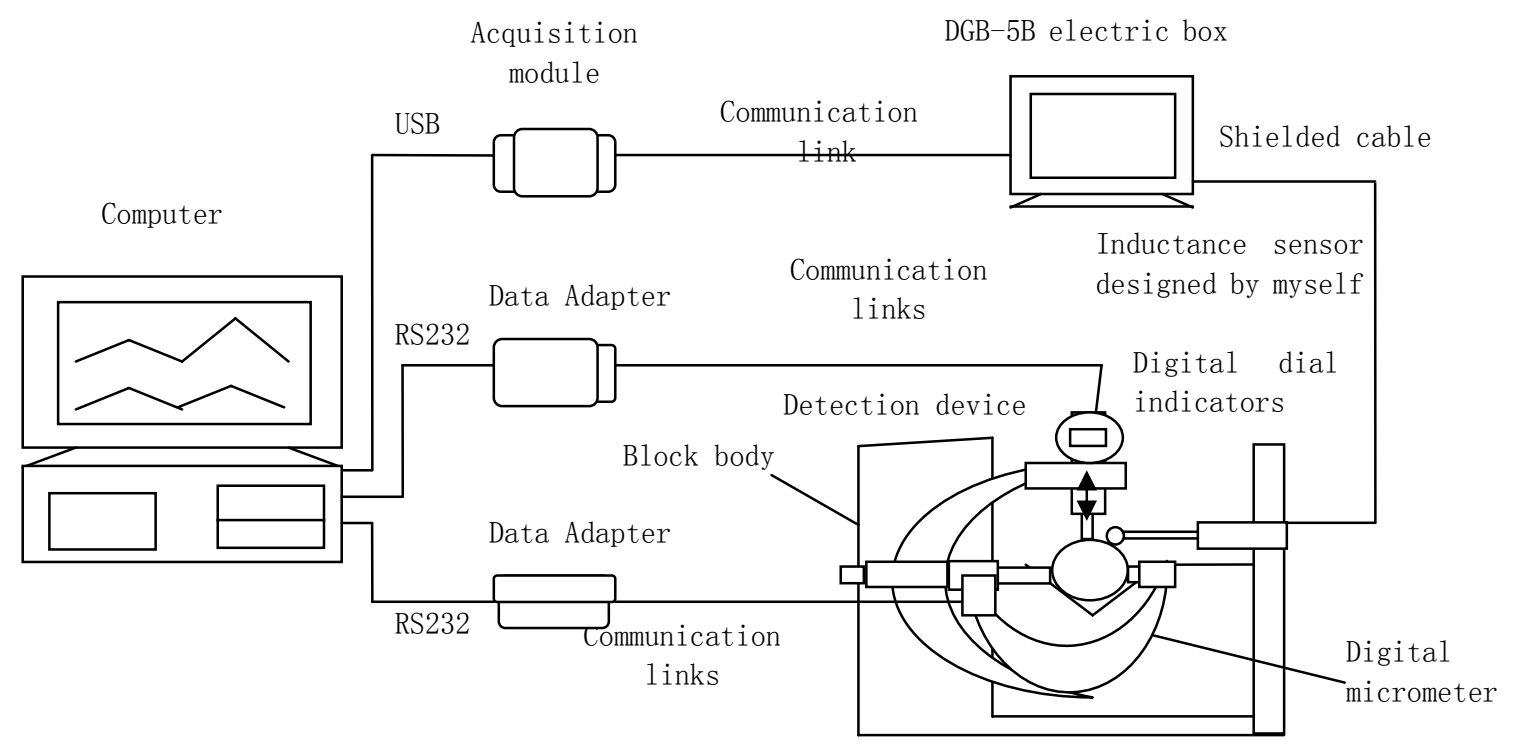

Figure 4. Combination solution of digital measurement technology 


\section{1) Digital detection technology}

Because part measurement has multiple dimensions, dimensional tolerances, shape and position tolerances needed to be measured, and its tolerance level is different, the measuring methods and instrument must be different, suitable measuring instruments have to be selected. The combined measurement program can be adapted to rotational shape part as the following: (a) Measuring of shape and position tolerance: the digital dial indicator and inductance micro meter are mainly used, the instrument' precise is 0.01 and 0.001 . (b) Measuring of dimension tolerances low 0.01(mm): Digital micrometer is used as the main measuring instruments, and the total

error value shall not be bigger than 0.006 (the max dimension is 100). (c) Measuring of dimension tolerances above 0.01: digital dial indicator and digital caliper are the main measuring instruments, and the total error value shall not be bigger than 0.03 (the max dimension is 300 ). A machining error detection system for rotational shape part is shown in Figure 4.

In machining precise detection system, if the condition of the enterprise is unfavorable, digital values of various measuring instruments can be read out in manual, and take it as the results of machining precision detection of rotational shape part; Enterprises, with good conditions, can link computers, instruments and acquisition part to automatically record inspection results

and save the records, print the inspection records, it also can achieve data communications with upper management systems.

2) Online interaction technology of quality data and information

In the past, quality data's remote interaction depended on disk, hard disk and manual records, it is not only inefficient, and financial resources, but also may not response the production quality situations in time. Distributed quality system under the condition of wireless network through the WEB and client programs can achieve interaction with online data and information, and guarantee its quickness and real-time.

\section{3) $C A Q$ mailbox technology}

In the distributed quality system under the condition of wireless network, in order to speed up information delivery, enhance the exchange of information, independent CAQ mailbox can be established.

4) Wireless networks construction technology

Wireless networks and broadband are a trend of information network, and the infrastructure of digital factory, and digital city. Treating infrastructure critically which have already existed, combining the old infrastructure with the new equipment, new technology in the infrastructure, to enhance the technical layer, improve services environments, lift operational efficiency, and ensure the quality of communication, are purpose and fate.

5) Data saving strategy and security technology

In order to keep the data and information not be leaked out, it is obligatory to enhance the system security management, it include setting safe account and passwords, using encryption technologies such as PIN encryption etc.

\section{CONCLUSION}

In order to introduce advanced techniques to quality system of large enterprises across the region, the distributed quality system under the condition of wireless network are designed, which can be divided into four layers: device layer, disposal layer, control and management layer, and decision-making layer. The core technology of distributed quality system include: digital detection technology, the quality data and information online interaction technology, CAQ mailbox technology, wireless network construction technology, data saving strategies and security technology. The network structure of the distributed quality system is supported by Internet technology, using Intranet as foundation, and Extranet as extension, and the wireless network achieves fully the wireless broadband interconnection.

\section{REFRENCES}

[1] GENG Hong qin, ZHANG Yuanying, Wireless Network and the Method of Building, Journal of Tianzhong, 2002, 5: 24-25.

[2] LIN Zhihang. The computer aided quality system [M]. Beijing, Mechanical Industry Press, 1996.

[3] Tan Rumou, R\&D of High Speed-Cutting-Machine, WMEM, 1999 (3), 8 .

[4] Wu Liaoyuan, Research on intelligent quality \& diagnosis technologies and the application in CIMS, 4, 2007.

[5] Zhang Gongxu, new quality management, high education press, May, 1998 